\title{
Barça-Madrid: una rivalidad global. Análisis del derbi a través de la prensa escrita española
}

\author{
Javier COTERÓN LÓPEZ \\ Universidad Politécnica de Madrid \\ j.coteron@upm.es \\ Mattew Fernando BELLO GARRIDO \\ Universidad Politécnica de Madrid \\ mattewbello@yahoo.com
}

Recibido: 23 de noviembre de 2011

Aceptado: 3 de julio de 2012

\section{Resumen}

La relación entre fútbol y medios de comunicación exhibe señales claras de interdependencia. La presente investigación se planteó determinar el tratamiento que los principales medios impresos españoles dieron a la información durante la cobertura de cuatro derbis Barça-Madrid en abril y mayo de 2011. Se realizó un análisis de contenido de los diarios El País, Marca, Sport y La Vanguardia, estudiando la caracterización del mensaje elaborado por cada uno de ellos y las influencias que sobre el discurso periodístico tienen factores extradeportivos, principalmente económicos y sociopolíticos. El estudio muestra los cambios que está experimentando la prensa escrita para cubrir un fenómeno de impacto global.

Palabras clave: Fútbol, medios de comunicación, mensaje periodístico, tratamiento de la información, Barça-Madrid.

\section{Barça-Madrid: a global rivalry. Analysis of the derby through the Spanish written press}

\begin{abstract}
The relationship between football and the media clearly shows interdependence signs. The aim of this study was to determine the treatment given by the major Spanish written media during the coverage of four Barça-Madrid derbies in April and May 2011. A content analysis of newspapers El Pais, Marca, Sport and La Vanguardia was conducted, studying the characterization of the message and the influences that on the speech-sporting journalism have certain factors, mainly economic and sociopolitical. The study shows the changes concerning the Spanish press in covering a global impact phenomenon.
\end{abstract}

Key words: Soccer, mass media, journalistic speech, information processing, Barça-Madrid.

Referencia normalizada: COTERÓN LÓPEZ, Javier y BELLO GARRIDO, Mattew Fernando (2012): "Barça-Madrid: una rivalidad global. Análisis del derbi a través de la prensa escrita española". Estudios sobre el mensaje periodístico, vol. 18, núm. 2 (julio-diciembre), págs.: 459-474. Madrid, Servicio de Publicaciones de la Universidad Complutense.

Sumario: 1. Introducción. 2. Deporte y medios de comunicación. 3. Un clásico global. 4. Metodología. 5. Análisis descriptivo; 5.1. Tratamiento periodístico; 5.2. Quién y de quién se habla. 6. Análisis de correspondencia; 6.1. Deportivos versus generales; 6.2. Centralismo frente a nacionalismo; 6.3. Maniqueísmo informativo; 6.4. Opinión para la confrontación. 7. Conclusiones. 8. Referencias bibliográficas.

\section{Introducción}

La simbiosis entre deporte y medios de comunicación es cada día más evidente. El primero se ha convertido en una gran fuente de contenidos y generador de audiencias de 
interés estratégico para los media (DE Moragas, 2000: 3), al tiempo que éstos, principalmente la televisión, son una de las principales fuentes de financiación de las entidades deportivas. El desarrollo de esta relación de interdependencia obedece al impacto que el deporte genera sobre la humanidad, trascendiendo sus propias fronteras y convirtiéndose en un fenómeno cultural, económico y político ampliamente reconocido por los estudiosos de las Ciencias Sociales (BlaIN y Boyle, 2002: 232).

Entre los deportes, el fútbol es el que más seguidores ostenta en todo el planeta. $\mathrm{Su}$ transformación, desde su inicio en las comunidades pudientes británicas a la manifestación global profesionalizada actual, ha hecho aumentar de forma exponencial el número de practicantes, competiciones, partidos y, sobre todo, aficionados. Semejante fenómeno ha contribuido a modificar el aspecto tradicional de la disciplina deportiva en aras de satisfacer las exigencias de la colectividad, dando pie a una dimensión espectacular.

España no ha escapado a este proceso y el balompié se ha convertido en el rey entre los deportes (LlOPIS, 2008: 56), al punto de ser considerado un signo de unificación sociocultural dentro de una sociedad cada vez más diversa. Como máxima expresión de esta característica, encontramos la rivalidad entre el Real Madrid Club de Fútbol (RMCF) y el Fútbol Club Barcelona (FCB), que en la actualidad ha adquirido un carácter universal.

Prueba de ello es la expectación creada con la celebración de cuatro partidos en apenas 18 días, entre finales de abril e inicios de mayo de 2011. La razón: una coincidencia de calendario que agrupaba en menos de tres semanas la disputa del partido de vuelta de la Liga de España, la final de la Copa del Rey y las semifinales de la Liga de Campeones de Europa. El primer choque estaba definido desde inicios de temporada, pero los tres restantes surgieron a partir del acceso de los clubes a las últimas fases de las respectivas competiciones.

Así, el prestigio deportivo traducido en títulos, los beneficios económicos obtenidos por los torneos y las motivaciones sociopolíticas surgidas por la rivalidad histórica entre Madrid y Barcelona se ponían en juego en un corto e intenso periodo de tiempo; el resultado de este enfrentamiento directo determinaría el éxito o fracaso de toda la temporada.

Evidentemente, los medios de comunicación se hicieron eco de la batalla, multiplicando la atención, posicionándose frente a los acontecimientos y llegando incluso a ser partícipes. Considerando estos factores, el presente estudio se planteó analizar el tratamiento que los principales medios impresos españoles dieron a la información en ese período; el objetivo es determinar el papel que jugaron en la contienda.

Para llevarlo a cabo se analizaron los contenidos referidos al Real Madrid y Barcelona en los diarios deportivos Marca y Sport, y los de carácter general El País y La Vanguardia, durante un período de tres semanas. Estos periódicos son los de mayor alcance de ventas en sus ciudades de origen, Madrid y Barcelona respectivamente, de acuerdo con el Estudio General de Medios (EGM, $2^{\mathrm{a}}$ oleada 2011). La dualidad, utilizada como criterio de selección en investigaciones previas (ISASI, 2006: 30), es premeditada, dada la influencia a la que se someten los medios de comunicación por su área geográfica más inmediata (AlCOBA, 2005: 83). 
La prensa escrita como soporte de información de carácter imperecedero (papel) con capacidad de cobertura al detalle, es una referencia necesaria y rigurosa como analista frente a la oferta de los medios audiovisuales (TÚÑEZ, 2010, 81).

\section{Deporte y medios de comunicación}

Deporte, lenguaje y cultura son elementos coincidentes dentro del desarrollo de la sociedad actual (MEÂN y HALONE, 2010: 254) y aunque sus interconexiones son múltiples, los investigadores apuntan hacia dos de especial relevancia: la identidad social e individual y los medios de comunicación.

En el primer caso, el deporte es situado como uno de los componentes que más contribuyen a afianzar la identidad colectiva de los pueblos, creando un sentido de pertenencia a la comunidad donde se insertan sus ciudadanos (BOYLE y HAYNES, 2002: 111). Su desarrollo como fenómeno sociopolítico en el último siglo lo ha convertido frecuentemente en herramienta al servicio de determinados intereses: por un lado, el nacionalismo ha utilizado los éxitos deportivos como bandera para sus fines proselitistas (RuIZ, 2009); por otro, las clases dominantes han incluido el deporte entre los mecanismos de control y poder sobre el resto de la población (BORDIEU, 1991: 361).

La explotación política del espectáculo deportivo y las consecuencias culturales y económicas de su desarrollo han derivado en la creciente globalización de los medios de comunicación y el deporte. Como soporte, el marketing y las nuevas tecnologías han generado la mejor relación posible entre medios, clubes, atletas, organizaciones y empresas para llegar a constituir en la actualidad un verdadero imperio del deporte (Rowe, 2004). Esta alianza comercial tiende a promocionar a deportistas y entidades como productos y favorecer su consumo en una sociedad cada día más demandante del deporte y todos sus componentes.

El periodismo deportivo, el más consumido y de mayor alcance social actualmente, ha alcanzado una dependencia extrema y peligrosa de los ingresos por publicidad, pues la "presunción de comercialidad" de un acontecimiento es un valor noticia que influye en la configuración de los contenidos de los medios de comunicación, lo que representa un arma de doble filo para la tarea periodística; y, por ende, para la calidad informativa (CRAIG, 2004: 234). Esta pérdida de autonomía puede llegar a ser realmente perversa por el carácter intrínseco de los medios como agentes formadores de opinión pública. Como apunta De Moragas (2007: 11), "la 'mediatización' tiene una especial influencia en la configuración de los valores culturales asociados al deporte". Éstos han cambiado en función de un modelo de exclusión al contrario, de limitación a la victoria y la derrota como expresiones de éxito o fracaso; en resumen, hacia una idea dicotómica de los protagonistas y los acontecimientos.

A todo esto se añade la tendencia actual a poner la información al servicio del entretenimiento, estimulando la banalización de los contenidos y sometiendo la veracidad a lo atractivo, a lo que divierte, o a lo que signifique más ventas mediante recursos publicitarios (DomínGUEZ, 2009: 4). Esta tendencia ha llegado a quitar al deporte gran parte de su protagonismo, supeditándolo a la concepción de espectáculo existente en los medios (Paniagua, 2006: 186). 


\section{Un clásico global}

En España, el RMCF y el FCB representan la más longeva e importante rivalidad deportiva, que ha saltado a otros ámbitos de la vida común con el paso de los años (CASTRO-RAMOS, 2008: 699), teniendo como resultado frecuentes incidentes dentro y fuera del escenario deportivo.

VÁZQUEZ MONTALBÁN (2005: 142) nos presenta la confrontación política y social de un RMCF-FCB, o viceversa, como "una válvula de escape de la irreconciliable apatía consensuada entre Madrid y Barcelona". Los madridistas han sido tildados (con razón o sin ella) de ser serviles a los propósitos del régimen franquista, aliados del Estado español centralista y promotores de la resistencia secesionista; mientras que los blaugranas buscan retomar su condición de ejército simbólico desarmado del nacionalismo catalán.

A pesar de las evidentes diferencias, ambos coinciden en haber desarrollado un gran poder económico y social que los convierte, más que en clubes de fútbol, en instituciones que buscan proyectar unos valores y una identidad corporativa de carácter universal. En ese sentido, la globalización de sus marcas salta a una esfera mediática de cuya dinámica los propios clubes son financieramente dependientes. No es casualidad que el ranking de clubes de fútbol más ricos del mundo 2011 de Football Money League, elaborado por Deloitte, lo encabecen el RMCF (con una facturación superior a las 438 millones de euros) y el FCB (con 398,1 millones). Esto obedece a la comercialización de productos y servicios, a la expansión del negocio hacia nuevos mercados como el asiático y a las ventajas del nuevo entorno digital, que ha permitido a éstas y muchas otras organizaciones deportivas desarrollar incluso sus propios medios de comunicación (GINESTA, 2010: 149).

En España, el llamado Clásico o Derbi figura cada año entre los programas más vistos de la televisión; de hecho, en 2011 la final de la Copa del Rey registró su audiencia más alta, superando los 14 millones de televidentes (Barlovento Comunicación). A nivel mundial, la rivalidad española sobrepasa con creces los mil millones de espectadores, convertido ya en un derbi globalizado.

GONZÁLEZ RAMALLAL (2004: 360) analizó a fondo esta dimensión espectacular del deporte en los medios españoles, constatando que "la información deportiva textual, verbal y visual distribuida por los medios de comunicación españoles más representativos configura una realidad deportiva predominantemente futbolística centrada en dos protagonistas principales: el RMCF y el FCB". Una realidad que desafortunadamente ha sido presentada por los medios en diversos momentos "como irreparable e innegociable; una rivalidad que no se encuentra en todos los eventos deportivos tipificados como deporte-espectáculo" (GINESTA, 2007: 154).

En lo deportivo, esta contienda ha tenido diversas etapas históricas caracterizadas por la supremacía de uno u otro equipo. En el momento coyuntural de este análisis el FCB goza de una superioridad incuestionable sobre sus rivales, enraizada en los valores de su cantera y en un estilo de juego que se ha convertido en referente del fútbol mundial; toda una afrenta para su contrincante, que necesita con urgencia revertir una situación que tiende a durar demasiado tiempo y dañar seriamente sus intereses. Para ello ha de poner todos los recursos disponibles al servicio de un fin: recuperar el primer lugar. 


\section{Metodología}

Para la realización de este estudio hemos analizado todos los artículos referidos a RMCF y FCB en los diarios Marca, Sport, El País y La Vanguardia publicados entre el 15 de abril y el 5 de mayo de 2011, correspondientes al período de celebración de los cuatro partidos. Durante este tiempo Marca publicó 21 ediciones, mientras que el resto editó 20 a causa de la jornada del Viernes Santo, día en que la mayor parte de los diarios no salen a la venta.

Es importante destacar la presencia intermedia de dos encuentros de Liga española ante otros rivales, que fueron incluidos en el estudio, pues forman parte del período de influencia de los Clásicos. Se excluyeron del análisis las portadas, así como todo tipo de imágenes (fotografías, infografías, gráficos, tablas estadísticas, etc.), limitando la revisión al texto escrito.

Una vez definida la muestra, realizamos un proceso de codificación de los datos a partir de un diseño preliminar de categorías (BARDIN, 2002: 78) que permitió delimitar las unidades de análisis en unidades de contexto (artículo periodístico) y de registro (párrafos, oraciones o ideas temáticas que presentan los artículos). Se generó una planilla de codificación inicial que, tras un estudio piloto y la colaboración de un investigador externo, quedó conformada en 9 categorías y 78 subcategorías.

Como unidades de contexto se incluyeron: Acontecimiento, Género periodístico y Fuente de información; mientras que como unidades de registro se contemplaron: Emisor, Referente, Valoración, Función del lenguaje, Tipo de lenguaje y Dimensión (temática).

El texto fue tratado con la ayuda del programa NVivo versión 8 para codificar cualitativamente los distintos fragmentos de cada artículo; tras la codificación, obtuvimos una tabla de frecuencias de categorías y subcategorías en cada uno de los periódicos con la que elaboramos el análisis descriptivo. Se eliminaron todos los códigos con un valor $\leq 5$ por considerarse atípicos de muy baja frecuencia, lo que redujo la cifra de subcategorías a 71 .

Dada la gran cantidad de unidades registradas, vimos la necesidad de reducir la complejidad de la información a través del análisis multivariante. Siguiendo la propuesta de CABALLERO y ViCENTE (2011), utilizamos el programa Multbiplot para, tratando los códigos como unidades taxonómicas o variables cualitativas, construir una matriz con la que realizar un Análisis Factorial de Correspondencias (AFC) (BENZÈCRI, 1976). El resultado es una representación gráfica en la que cada subcategoría y cada periódico son representados en el plano factorial por un marcador, posicionado en función de las distancias relativas al resto de marcadores. La combinación de categorías y periódicos en un mismo plano indica la correspondencia entre ambos. Para facilitar la identificación de estas relaciones, realizamos un análisis cluster; procedimiento que une los distintos códigos en torno a un centro de gravedad. La distancia con dicho centro indica la correspondencia específica de las subcategorías con los periódicos, pudiéndose inferir a partir de su agrupamiento la caracterización específica de cada medio.

Para interpretar los resultados y poder realizar las inferencias pertinentes a lo largo de todo el estudio, empleamos el análisis de contenido, definido por Klaus KRIPPEN- 
DORFF (2002: 28) como una "técnica de investigación destinada a formular, a partir de ciertos datos, inferencias reproducibles y válidas que pueden aplicarse a su contexto", siendo considerados los datos "como manifestaciones simbólicas o indicadores de los fenómenos".

\section{Análisis descriptivo}

Tras una revisión exhaustiva de los textos, codificamos 20939 unidades de análisis (registro y contexto), dentro de los 1428 artículos dedicados al objeto de estudio (ver Tabla 1). La superficie dedicada a RMCF y FCB representó el 41,96\% del total de informaciones deportivas publicadas por los diarios durante las tres semanas que rodearon los cuatro clásicos. Esto es una prueba de la importancia mediática del fenómeno.

Tabla 1. Datos totales de análisis

$\begin{array}{cccc}\text { Diario } & \begin{array}{c}\text { Unidades de } \\ \text { análisis }\end{array} & \text { Artículos } & \begin{array}{c}\text { Páginas dedicadas } \\ \text { al RMCF-FCB }\end{array} \\ \text { Marca } & 1929 & 544 & 37,90 \% \\ \text { E1 Pais } & 2778 & 104 & 50,97 \% \\ \text { La Vanguardia } & 7743 & 163 & 59,05 \% \\ \text { Sport } & 8489 & 617 & 41,52 \% \\ \text { TOTAL } & \mathbf{2 0 9 3 9} & \mathbf{1 4 2 8} & \mathbf{4 1 , 9 6 \%}\end{array}$

La temática predominante, como no puede ser de otro modo, se ciñó al aspecto deportivo $(74,77 \%)$. Sin embargo, cabe destacar la relevancia que adquirieron los asuntos institucionales $(9,58 \%)$, surgidos de la polémica generada por hechos puntuales sobre el terreno de juego. La expulsión de un jugador sirvió para desencadenar una sucesión de acusaciones que, en muchos casos, eclipsaron lo puramente deportivo: "El FC Barcelona anunció anoche que sus servicios jurídicos estudiarán si las declaraciones que realizó anoche José Mourinho sobre las supuestas ayudas arbitrales pueden incurrir en alguna falta a los reglamentos de la UEFA y, en ese caso, recurrir a su comisión de disciplina" (La Vanguardia, 28/04/11: 52).

En todos los periódicos predomina la utilización de un tipo de lenguaje natural (79,18\%) que, junto con el coloquial $(14,92 \%)$, caracterizan la práctica totalidad de los escritos. El lenguaje literario, de carácter casi residual (2,66\%), triplica su presencia en los periódicos generales respecto a los deportivos. Al realizar el análisis del peso relativo que esta información suponía en cada periódico, obtuvimos otro dato de interés que se verá reforzado en el análisis de correspondencias: los periódicos catalanes lo utilizaron en mayor medida, mientras que Marca salió mal parado en la comparación.

\subsection{Tratamiento periodístico}

Como refleja el Gráfico 1, la noticia (89,90\%), por su esencial carácter informativo, supera de forma amplia al resto de los géneros periodísticos en las publicaciones deportivas. En los diarios generales predomina la tendencia hacia el comentario o crítica (64,32\%), un género híbrido entre la información y la opinión (interpretación), muy común dentro de la redacción deportiva, pues sirve "para dar a conocer juicios valorativos" (MARTínez ALBERTOS, 2000: 352).

La opinión, representada a través de la columna y los editoriales, es ampliamente superior en los periódicos deportivos, con el atenuante de que sus editoriales son de- 


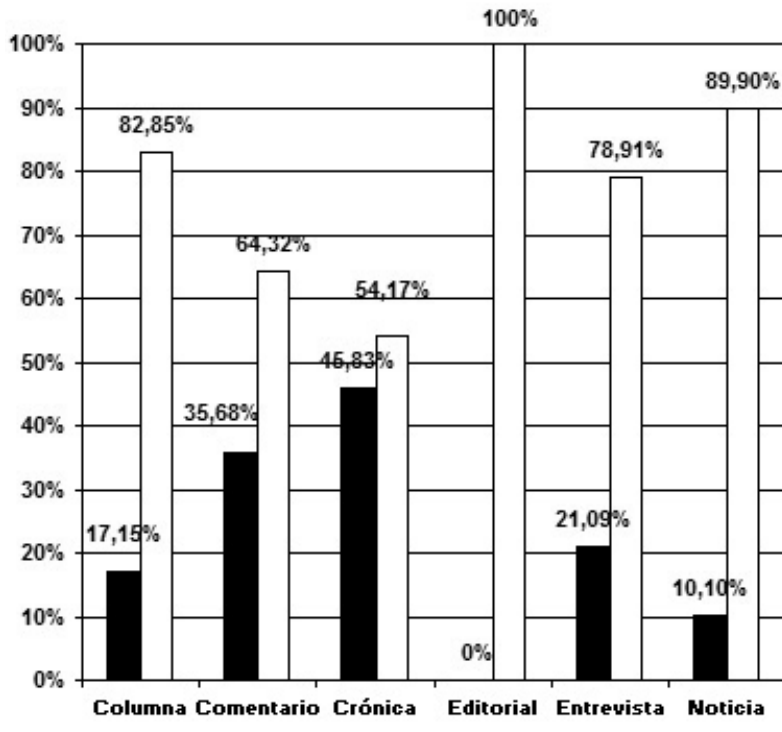

Gráfico 1 . Géneros periodístic os de acuerdo al tip o de diarios Fuente: Coterón y Bello, 2011 dicados de forma exclusiva al fenómeno estudiado. La opinión crítica hacia determinados temas revela el claro posicionamiento de los diarios hacia un equipo u otro: "porque es de eso de lo que estamos hablando, de fútbol, y del mejor equipo del mundo, el Real Madrid" (Marca, 30/04/11: 47).

Un género casi inexistente en los documentos estu-

diados es el reportaje (al punto de considerarse valor atípico), lo que evidencia un desinterés hacia la investigación en profundidad por parte de los periódicos. Esta tendencia, popularizada en los últimos años, ya había sido comprobada por GonZÁLEZ RAMALLAL (2004: 261): "la prensa deportiva española tiende a centrarse en mayor medida en el dato, en la anécdota, que en el pausado y más profundo análisis en forma de reportaje o informe periodístico sobre un acontecimiento deportivo" y refleja la progresiva concepción de este deporte como un espectáculo de consumo.

\subsection{Quién y de quién se habla}

La gran mayoría de los artículos de prensa son responsabilidad de los miembros o colaboradores de la redacción, a quienes se atribuye el $63,26 \%$ de la autoría de los contenidos. El resto de la información aparece reflejada, a modo de citas textuales, con declaraciones de terceros $(12,20 \%)$ o de los miembros de los clubes $(24,54 \%)$. En el análisis

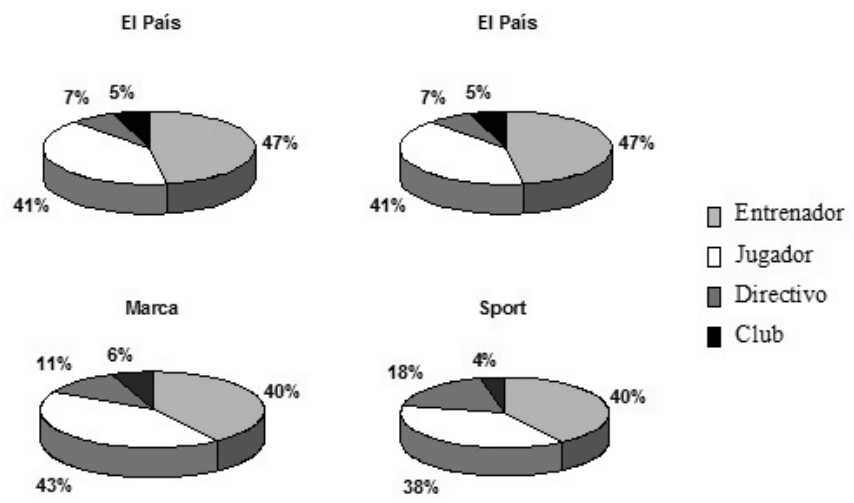

Gráfico 2. Quién habla en los clubes

Fuente: Coterón y Bello, 2011. 
particular de estos últimos resalta la figura de los entrenadores $(10,59 \%)$ sobre los jugadores $(9,66 \%)$, comprobando el protagonismo que estos personajes han adquirido en los últimos años (ver Gráfico 2).

En cuanto a los referentes (aquellos de los que se habla), las plantillas de cada equipo, tipificadas en los textos como conjunto o estilo de juego, son las más aludidas $(21,30 \%)$, no evidenciándose especial predominancia de una sobre otra. Donde sí observamos diferencias significativas es en la información dedicada a los individuos (ver Gráfico 3). En el FCB los jugadores, asociados principalmente a referencias de trabajo y actitud, son objeto de mayor dedicación informativa que su entrenador; sin embargo, la tendencia se invierte en el RMCF, donde el técnico acapara el foco mediático.

Si hacemos un análisis global de la situación, los entrenadores hablan más, y se habla más de los entrenadores; una situación novedosa que aparece en los últimos años, invirtiendo la situación existente con anterioridad, como corrobora el estudio longitudinal realizado por ISASI (2006: 113).

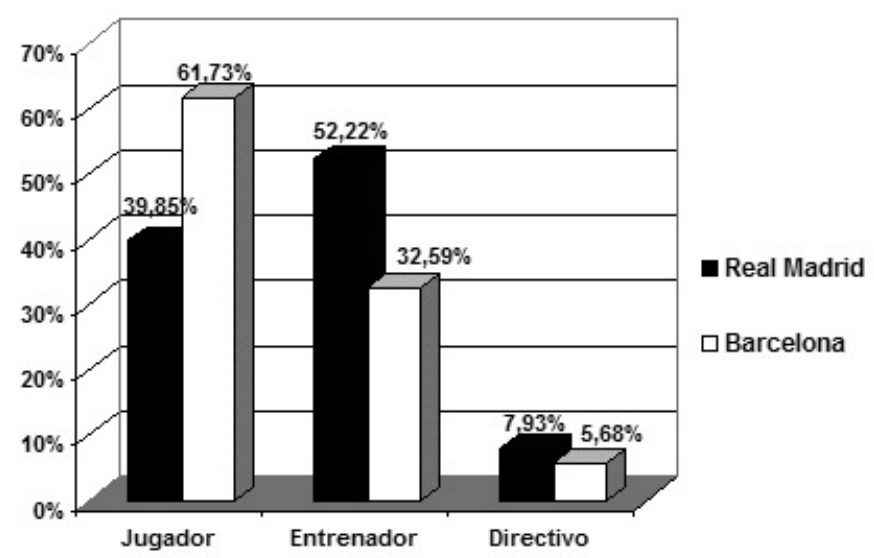

Gráfico 3. Referentes por clubes

Fuente: Coterón y Bello, 2011

En nuestro estudio, Mourinho destaca como referente por encima de Guardiola; sin embargo, el entrenador culé es quien más veces aparece declarando; una situación que bien podría deberse a que Aitor Karanka, asistente de Mourinho, fue designado para hablar en lugar del portugués en las ruedas de prensa de dos de los cuatro partidos, lo que no fue bien recibido por los medios de comunicación. El papel mediático que asumen los técnicos está modificando la imagen que proyectan los clubes y la valoración que se hace de ellos: "El presidente del Comité Olímpico Español, Alejandro Blanco, le dio un toque de atención a José Mourinho al decir que 'ha echado por la borda todos los valores que representa el club' y lamentó que las palabras del entrenador hubieran empañado el partido..." (Sport, 29/04/11: 5)

El RMCF fue percibido negativamente en mayor medida que el FCB en todos los diarios, incluidos los de Madrid: "Frente a un Madrid otra vez encogido y acuartelado, el equipo azulgrana empinó la semifinal a su manera, con mayor gusto y decisión que su adversario..." (El País, 28/04/11: 47). Esta cita también demuestra la preferencia de los medios a valorar positivamente al FCB y todos sus integrantes, a excepción del diario Marca, que se decanta por hacer un juicio más halagador hacia el RMCF. 
Los diarios toman partido a la hora de informar: un 59,05\% de los textos benefician o desfavorecen a sus referentes, mientras que los juicios neutrales aparecen solo en un $25,52 \%$ del discurso. El resto, categorizados como ambivalente $(15,43 \%)$, se agrupan en dos tipos de discurso: el ambiguo y el irónico. El primero se utiliza principalmente para no tomar partido en momentos delicados: "un Barça que lo tuvo todo en su mano, pero que no perdió ni un ápice de confianza para afrontar la final de Copa del miércoles" (La Vanguardia, 17/04/11: 60); y el segundo, que adquiere formas que van desde lo humorístico hasta lo cruel, llegando en ciertos momentos a crear suspicacias: "Creía que era odio, pero empiezo a pensar que es amor. Te necesito, Madrid. Te necesito para noches como la de ayer, en las que consigues llenar de felicidad a los culés" (La Vanguardia, 04/05/11: 52). Esta figura retórica es común en el periodismo deportivo para criticar temas o individuos como parte de un mensaje latente.

\section{Análisis de correspondencia}

El establecimiento de clusters en el AFC nos permite observar la existencia de tres agrupaciones de subcategorías que caracterizan a los periódicos próximos (ver Gráfico 4). El País y La Vanguardia comparten la caracterización del cluster 1, Marca el cluster 2 y Sport el cluster 3. Sus principales características (subcategorías) aparecen reflejadas en la Tabla 2, con los respectivos códigos.

TFC: Tratamiento periodistico

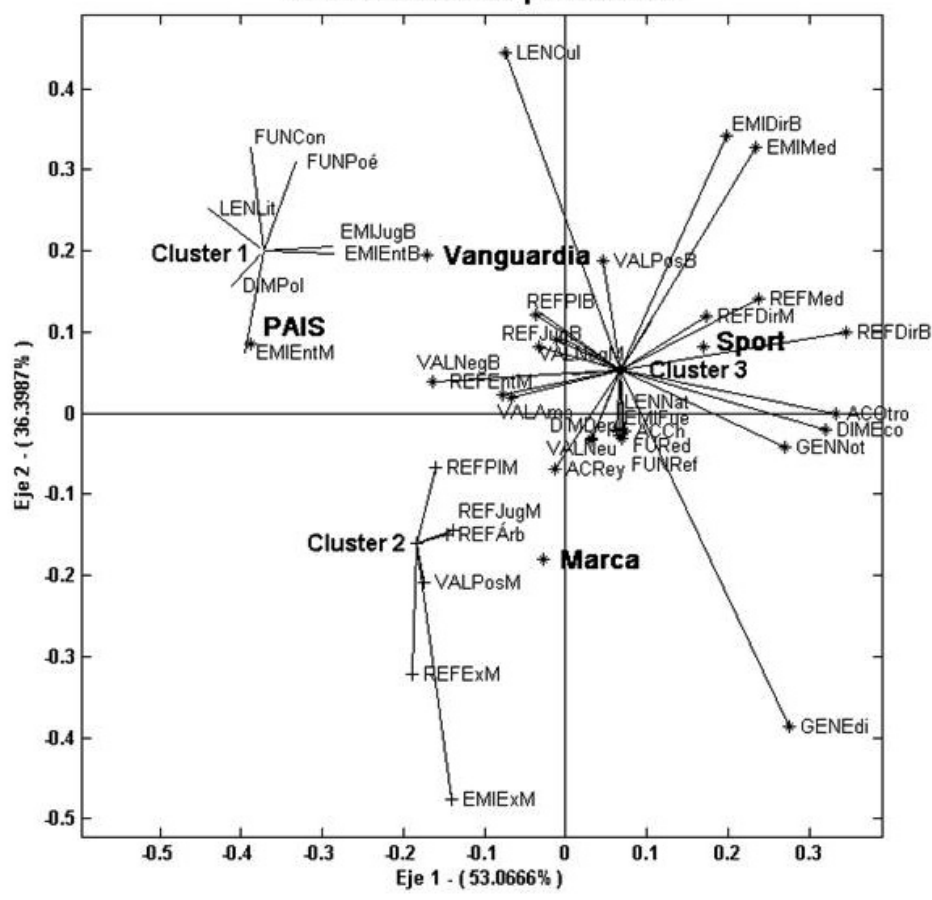

Gráfico 4. Análisis factorial con clusters

Fuente: Coterón y Bello, 2011 


\subsection{Deportivos versus generales}

La cercanía de El País y La Vanguardia en el plano (cluster 1) denota ciertas características comunes que los definen como los diarios generales que son. Más sobrios y sosegados en sus textos, ceden la palabra principalmente a los protagonistas: jugadores y entrenadores. Este es un factor diferenciador respecto a los diarios deportivos, que recurren más a fuentes externas o a sus propias declaraciones; en muchos casos cargadas con un alto grado de opinión. Sport (cluster 3), utiliza este género periodístico para defender lo propio y atacar lo ajeno, mientras que Marca (cluster 2) se centra en la defensa de su club de referencia.

El exceso de opinión es una práctica nociva que tienden a utilizar los medios de comunicación como parte de un estilo sensacionalista, que solo persigue la obtención de mayores beneficios económicos: "Para la prensa de información general no existen noticias, salvo hechos muy excepcionales, que incrementen considerablemente la venta; en la deportiva, los triunfos del Madrid y del Barcelona se traducen en la adquisición de miles de ejemplares" (GARCía CANDAU, 1996: 230).

Un medio para llamar la atención y atraer las ventas es precisamente el lenguaje. Mientras la prensa general emplea un vocabulario más literario, culto o científico, la deportiva se vale de un lenguaje coloquial y/o natural, con el que se busca acercar al lector a quien expone las ideas; sin embargo, a veces se sobrepasa el límite y brota el lenguaje vulgar, que por sí mismo se vuelve fuente de información: "En esta sala, el puto jefe, el puto amo, es él. Es el que más sabe del mundo. Yo no quiero competir ni un instante" (Josep Guardiola, El País, 27/04/11: 47).

\begin{tabular}{|c|c|c|c|c|c|}
\hline Cluster 1 & Código & Cluster 2 & Código & Cluster 3 & Código \\
\hline Género comentario & GENCom & Referente Jugador RMCF & REFJugM & Lenguaje Culto-Científico & LENCu \\
\hline Género crónica & GENCro & Referente Plantill a RMCF & REFPIM & Valoración A mbivalente & VALAmb \\
\hline Emisa FCB & BMICIB & Valoración positiva RMCF & VALPosM & Valoración Negativa RMCF & VALNegM \\
\hline Emisor Entrena dor $\mathrm{FCB}$ & EMIIEnB & Emisor Directivo RMCF & EMIDirM & Valoración Positiva FCB & VALPosB \\
\hline Emisor Entrenador RMCF & EMIIEnM & Emisor Aficiona dos RMCF & BMIAfM & Referente Plantilla FCB & REFPIB \\
\hline Bmisc Jugad $\propto$ FCB & BMIUgB & Emisor RMCF & BMCIM & Emisor Fuente de informac. & EMIIFve \\
\hline Función poética & FUNPoé & Emisor otro & EMIOtro & Dimensión Económica & DIMEco \\
\hline Lenguaje literario & LENL it & Género columna & GENCol & Referente Club FCB & REFCIB \\
\hline Dimensión politica & DIMPol & Lenguaje volgar & LENVu & Género Editorial & GENEdi \\
\hline
\end{tabular}

Tabla 2. Principales subca tegorías de análisis agrupadas por clusters

\section{Fuente: Coterón y Bello, 2011}

El discurso generado en torno al RMCF tiende a utilizar figuras más informales, mientras que el conectado al FCB se vale de construcciones más elegantes y elogiosas: "Equiparar las falsedades, y alguna que otra memez, de Mourinho a las contadas y argumentadas exposiciones de Pep parece un trabajo demasiado complicado para la guardia pretoriana blanca" (Sport, 25/04/11: 8).

Además del lenguaje, la temática ilustrada es también comparable entre ambos formatos de prensa. Por encima de la mayoritaria dimensión deportiva, los diarios deportivos dan más relevancia a asuntos de tipo económico, como los beneficios financieros de los clubes o los costes de los posibles fichajes; al tiempo que los impresos de corte general apuntan más hacia la política, aprovechando para filtrar ideologías y/o líneas editoriales: "El Barça se sobrepuso a todo, a todas las batallas, la mayoría extradeportivas, como la del catalán" (La Vanguardia, 28/04/11: 49). Esta realidad se sustenta en mayor medida al comparar los rotativos en función de su origen. 


\subsection{Centralismo frente a nacionalismo}

Al trasladar los razonamientos hacia el ámbito geográfico, detectamos la ya histórica lucha del centralismo ejercido por Madrid contra el reclamo independentista expresado por Barcelona. Ya hace algunos años, GARCíA CANDAU (1996: 230) afirmaba que "hasta para la compra de periódicos hay militancias. Los periódicos deportivos actuales han de ser forzosamente madridistas o barcelonistas. Otro producto no es comercial".

La prensa de cada región se hace eco de las proclamas propias de su lugar de procedencia, que pasan además por contrarrestar las consignas del contrario: "Se quiere evitar que se estropee este momento y tenga más protagonismo cualquier tipo de manifestación contraria a nuestro himno" (Marca, 19/04/11: 2).

Los medios catalanes optaron no solo por enarbolar su bandera reivindicativa, sino también por atacar a sus pares madrileños por la postura protectora hacia el RMCF y las acusaciones en contra del FCB: "Sumergido durante 48 horas en las profundidades de la caverna mediática (conocida ahora también como 'central lechera') he podido comprobar cómo el madridismo ha pasado de la euforia a la decepción sin solución de continuidad gracias a los dos golpes mortales asestados por Guardiola y Messi" (Sport, 19/04/11: 13).

Este tipo discurso, aunque sea de corte negativo y peyorativo, va en concordancia con el principio geográfico de la información que relata AlCOBA (2005: 83), y que en el caso de Marca se acentúa con la nominación mayoritaria de personas relacionadas al RMCF. Su postura hacia los temas del club de la capital fue ampliamente favorable, en contraposición con El País, que a pesar de editarse en Madrid, mostró una actitud más crítica y muy relacionada con el discurso y la actuación de José Mourinho: "¿Comparte la cúpula del Real Madrid los criterios de su entrenador, José Mourinho? O, para plantearlo de otra manera: ¿Han caído todos en la locura o queda en el Bernabéu algún rayo de luz?" (El País, 01/05/11: 54).

Como ya mencionamos en el análisis de frecuencias, la figura del técnico portugués despierta controversia en las opiniones manifestadas por todos los diarios, llegando incluso a ser más mencionado en Barcelona, donde es percibido como un agente perjudicial para su club y para el fútbol en general: "El Real Madrid de José Mourinho, enemistado con casi todas las aficiones de la Liga por sus turbios procedimientos, el mismo Madrid que ha convertido en costumbre la ofensa y el pisotón, se presenta ahora como abanderado del juego limpio" (La Vanguardia, 03/05/11: 65).

Los diarios catalanes enarbolaron la bandera de los valores como elemento comparativo, presentando a un RMCF inmerso en una espiral de pérdida de identidad provocada por la ansiedad: "Pero lo que debe valorar el Madrid, ese Madrid que siente orgullo cada vez que habla de sus nueve Copas de Europa y de ser uno de los clubes más laureados del mundo, es que su imagen, su prestigio y su política de comunicación está bajando en picado (La Vanguardia, 16/04/11: 54); y al club culé como referente de un señorío moral: "Es por ello que llegado este martes, el Barça, su equipo, la 'gent blaugrana' han de dar ejemplo y acabar con este acoso con el 'seny' que les caracteriza" (Sport, 02/05/11; 64). En el intercambio de golpes, la prensa barcelonesa mantuvo un lenguaje más refinado y cuidado. 
Donde sí hubo más acuerdo entre los medios fue en la valoración de los árbitros, siendo calificados mayoritariamente de forma negativa; y con más intensidad por parte de Marca, que se hizo solidario con las persistentes quejas de los madridistas: "Stark golpeó al Madrid y De Bleeckere lo remató. El colegiado belga cumplió con las peores expectativas de Mourinho" (Marca, 04/05/11: 4).

\subsection{Maniqueísmo informativo}

Desde hace tiempo FCB y RMCF copan la información deportiva en los medios españoles. El pulso informativo se mantiene gracias a la aparición de hechos que reavivan cada cierto tiempo la rivalidad latente. Sin embargo, como hemos ido reflejando, esta confrontación tiende a materializarse últimamente en torno a ciertas personas y su carisma: Mourinho versus Guardiola o Messi frente a Cristiano, son dos claros ejemplos.

El excesivo protagonismo que vienen tomando estas figuras, ya sea por razones comerciales (jugadores) o ideológicas (entrenadores), ha llevado a configurar la redacción de los textos prácticamente alrededor de su estampa: "Viene esto a cuento por la mala prensa, española especialmente, del entrenador del Madrid, José Mourinho, y la buena, ganada a pulso, de Pep Guardiola, el del Barcelona" (El País, 30/04/11: 53).

Esta tendencia, cada vez más acentuada en el fútbol moderno, ha elevado las responsabilidades de los protagonistas por alcanzar el liderazgo en su parcela: mejor jugador del mundo o mejor pagado, mejor entrenador o más mediático. La pugna por el liderazgo entre Mourinho y Guardiola despierta el interés mediático hasta el punto de constituirse en objeto de estudio. Cubeiro y Gallardo (2010: 200) sugieren que este fenómeno se debe a que ambos técnicos "cultivan una identidad propia" en virtud de factores como fidelidad a las ideas, imagen, y una ingeniosa expresividad, puesta a prueba en las ruedas de prensa.

Esta visión dicotómica ha trascendido el valor de lo personal -mejor jugador o entrenador- para impregnar todo el discurso periodístico y no dejar lugar a terceras interpretaciones, haciendo un flaco favor al equilibrio informativo: "Era como si en vez de los dos grandes del fútbol mundial, el equipo barcelonista se estuviera enfrentando con un modesto, con un conjunto del montón, con la diferencia que el Madrid cuenta con excelentes defensas y con muy buenos delanteros" (La Vanguardia, 17/04/11: 61).

Con este enfoque limitado, el redactor busca promover su idea, intentando mantener la fidelidad del lector con un discurso análogo a sus intereses o aficiones, aunque las afirmaciones se sustenten en tesis más próximas al fanatismo que a la racionalidad, tomadas de situaciones puntuales y no en el seguimiento de hechos o conductas sostenidas en el tiempo. Así, en apenas una semana de separación, Marca elevó al RMCF por su triunfo en la Copa del Rey, y luego lo condenó por su derrota en la ida de semifinales de Liga de Campeones:

"La victoria despeja muchas de las urgencias del Madrid sometido al gobierno del Barça durante los dos últimos años, con algunas derrotas que dejaron heridas muy profundas en el madridismo" (Marca, 21/04/11: 2).

"La fugaz naturaleza del fútbol actual se manifestó con toda su crudeza en el Bernabéu. El Madrid entró al partido entre gritos de ‘icampeones, campeones!' y salió con el prestigio por los suelos" (Marca, 28/04/11:3). 
La visión particular de cada medio hacia hechos específicos responde a intereses que sobrepasan la naturaleza de la propia competición. "Madrid y Barcelona van de la mano en lo que a los favores de la prensa se refiere... ninguno de ambos clubes necesita comprar voluntades; se le ofrecen espontáneamente" (GARCÍA CANDAU, 1996: 230). Como ejemplo, se presenta la postura de cada periódico hacia el árbitro tras la expulsión del jugador del RMCF Pepe, en un partido de Liga de Campeones de Europa:

"El colegiado fue quizá demasiado riguroso" (El País, 28/04/11: 48a).

"Tuvo que ser Wolfgang Stark quien frenara en seco con la tarjeta roja las delicadezas del central portugués" (La Vanguardia, 28/04/11: 50).

"No estuvo muy acertado el alemán Stark, aunque, como fan de Messi, no me negarán que tiene que estar más que contento" (Marca, 28/04/11: 10a).

"Pepe vio la roja directa por una entrada criminal sobre Dani Alves a la altura de la rodilla" (Sport, 28/04/11: 5).

La variedad de interpretaciones bien puede responder al criterio individual del emisor; sin embargo cada frase responde al carácter específico del medio al que se circunscribe.

\subsection{Opinión para la confrontación}

El comprobado exceso de opinión de los medios se encargó de caldear los ánimos y avivar la polémica tanto dentro como fuera del terreno de juego. En su discurso, los propios entrenadores fomentaron la lógica tensión que traen consigo cuatro derbis: "El hecho es que ellos tienen una cosa muy difícil de conseguir, que es el poder. ¿Por qué en un partido equilibrado, que está para 0-0, el árbitro ha hecho esto?" (José Mourinho, El Pais, 28/04/11: 50).

Este argumento, como muchos otros, motivó la sobreexcitación de una afición ya bastante alterada. La hostilidad se hizo presente entre todos los estamentos de los clubes estudiados y las históricas relaciones entre el RMCF y el FCB volvieron a verse cuestionadas: "las tensiones de la última semana provocadas por el cruce de demandas entre los dos clubes ha enfriado la relación, como mínimo a nivel institucional,..." (Sport, 03/05/11: 25).

Los periódicos también formaron parte de un conflicto que nunca les perteneció, pero que se encargaron de acrecentar. Salieron en defensa de los suyos y, en muchos casos, atacaron al contrario con el argumento popular español del "y tú más": "Resulta que ahora es el propio entrenador del Barça el que vende jarabe victimista, al que acompaña con bromitas sobre el césped del Bernabéu, ironías respecto a un posible arbitraje luso o peculiares análisis sobre la riqueza y profundidad del banquillo blanco" (Marca, 25/04/11: 54). "Pero Mourinho debería entender que el fútbol es un deporte en el que no ganan los tramposos, los tahúres, ni los mentirosos. El grado de tensión que el portugués ha generado esta temporada..." (La Vanguardia, 28/04/11: 55).

\section{Conclusiones}

El fenómeno social que implica un Barça-Madrid trasciende lo meramente deportivo, convertido en un fenómeno de carácter espectacular con múltiples connotaciones que 
influyen en labor periodística de los medios españoles. De forma aislada, un partido ya representa una batalla épica digna de especial cobertura. Al converger de manera casual cuatro compromisos de este tipo en tan corto período de tiempo, los periódicos han volcado su atención para ofrecer la mayor cantidad posible de detalles, en un esfuerzo por mantener el pulso informativo. De esta forma, la mera calidad informativa se ha visto condicionada por factores que, partiendo de los simples hechos sucedidos en el terreno de juego, precisaban de una amplificación mediática para mantener la atención de los lectores.

Como se ha podido comprobar, los diarios estudiados, representativos de las sociedades madrileña y catalana, dedican parte de sus páginas a mostrar diversas aristas de un evento deportivo que al final fue juzgado bajo una visión reducida y limitada de los hechos. El mensaje apunta más a satisfacer afinidades que a hacer valoraciones equilibradas.

A excepción de El País, que exhibe un tratamiento más ecuánime y ponderado de la información, Marca, La Vanguardia y Sport optan por abordar el acontecimiento con una fuerte carga ideológica, tomando partido en la valoración de hechos y personas. Los juicios particulares invaden los espacios dedicados a la información, creándose una matriz de opinión que ha colaborado a exacerbar los ánimos entre jugadores, clubes y aficionados; y hasta entre los propios medios. Nuevamente, las diatribas históricas entre Madrid y Barcelona se intentaron dirimir sobre el césped, pero la confrontación sobrepasa este nivel, ayudada en este caso por la labor de la prensa escrita.

La defensa exagerada de lo propio y la descalificación del contrario han sido armas comunes dentro de un mensaje ideológico con fuertes connotaciones comerciales. La pérdida de terreno de los periódicos ante las nuevas plataformas digitales de información les ha llevado a mantener un discurso parcializado, lleno de tópicos y de apreciaciones dirigidas a contar todo en términos absolutistas, de blanco o negro; no en vano, han sido los mismos medios los que han fomentado el protagonismo de personajes como Mourinho y Guardiola, hasta el punto de convertirlos en figuras casi más trascendentes que los propios clubes, a sabiendas de que sus declaraciones o acciones garantizan futuras ventas.

Como se ha podido demostrar, el mensaje periodístico está cambiando en el tratamiento de los acontecimientos, condicionado fundamentalmente por el carácter espectacular y mediático que está adquiriendo el deporte, convertido en un objeto de consumo para grandes sectores de la población. Estos cambios se caracterizan por la magnificación del detalle, la focalización en determinados actores más allá del terreno de juego y la búsqueda de eslóganes que capten la atención de los espectadores y aumenten las ventas. Las atribuciones tomadas por la prensa escrita han ido en detrimento de la labor imparcial y equilibrada que deben tener los medios de comunicación.

En el momento álgido de la disputa, al borde de la ruptura institucional, un nuevo actor muy relacionado con el FCB y el RMCF ha saltado a la palestra: la selección española de fútbol, que en la actualidad está formada mayoritariamente por jugadores de ambos conjuntos. Ante el nivel de crispación provocado por la acumulación de encuentros y la sobrecarga de información, surge la figura del seleccionador nacional, quien reclama de los actores el fin de la confrontación y la recuperación de la unidad 
por los posibles daños colaterales que los derbis puedan dejar sobre el combinado nacional: "Los jugadores que no se hagan acreedores de nuestra confianza o trasladen a la Selección el mal ambiente reinante, tendrán que ser consecuentes con sus actos y serán sólo ellos los responsables de ganar o perder el privilegio de pertenecer a la plantilla de la Selección" (Vicente Del Bosque, Marca, 04/05/11: 25).

\section{Referencias Bibliográficas}

ALCOBA LÓPEZ, Álvaro (2005): Periodismo deportivo. Madrid, Síntesis.

BARDIN, Laurence (2002): El Análisis de Contenido. Madrid, Akal.

BENZÈCRI, Jean-Paul (1976): L'Analyse des Donées: L'Analyse des correspondences. Paris, Dunod.

BLAIN, Neil y BOYLE, Raymond (2002): "Sport as real life: Media, sport and culture", en BRIGGS, Adam y COBLEY, Paul: The Media: An introduction. Edimburgo, Pearson Education Limited, pp. 415-426

BORDIEU, Pierre (1991): "Sports and Social Class", en MUKERJI, Chandra y SCHUDSON, Michael: Culture. Contemporary perspectives in cultural studies. Londres, University of California Press, pp. 357-373

BOYLE, Raymond y HAYNES, Richard (2002): "New Media Sport", en Sport, media, culture, $\mathrm{N}^{\mathrm{o}}$. pp. 95-114.

CABALLERO JULIÁ, Daniel y VICENTE GALINDO, María Purificación (2011): "El HJ-Biplot como Herramienta en el Análisis de Grupos de Discusión". Salamanca, Universidad de Salamanca.

CASTRO-RAMOS, Elga (2008): "Loyalties, commodity and fandom: Real Madrid, Barça and Athletic fans versus 'La Furia Roja' during the World Cup", en Sport in Society, $\mathrm{N}^{\mathrm{o}}$ 11(6). pp. 696-710.

CRAIG, Robert (2004): "Business, Advertising, and the Social Control of News", en Journal of Communication Inquiry, $\mathrm{N}^{\mathrm{o}}$ 28(3). pp. 233-252.

CUBEIRO, Juan Carlos y GALLARDO, Leonor (2010): Mourinho versus Guardiola: Dos métodos para un mismo objetivo. Barcelona, Alienta Editorial.

DE MORAGAS, Miquel (2000): “Olimpismo, comunicación y cultura", en http://olympicstudies.uab.es/pdf/wp095_spa.pdf. [fecha de consulta: 20 de marzo de 2011]

DE MORAGAS, Miquel (2007): "Comunicación y deporte en la era digital", en http://olympicstudies.uab.es/pdf/wp107_spa.pdf. [fecha de consulta: 15 de marzo de 2011]

DOMÍNGUEZ PORTELA, Javier (2009): "Ensayo sobre la cuádruple espectacularidad del periodismo deportivo", en Razón y palabra, No 69 , pp. 1-15.

ESTUDIO GENERAL DE MEDIOS (2011): AIMC octubre de 2010 a mayo de 2011. [fecha de consulta: 5 de julio de 2011] 
GARCÍA CANDAU, Julián (1996): Madrid-Barça: historia de un desamor. Madrid, El País Aguilar.

GINESTA PORTET, Xavier (2007): "Los valores en el deporte: una experiencia educativa a través del Barça-Madrid", en Comunicar: Revista científica iberoamericana de comunicación y educación, $\mathrm{N}^{\mathrm{N}} 28$. pp. 148-156.

GINESTA PORTET, Xavier (2010): "Los medios propios de los clubes de fútbol españoles. De la revista oficial a los canales de TDT", en Estudios sobre el Mensaje Periodístico, vol. 16, pp. 145-166.

GONZÁLEZ RAMALLAL, Manuel (2004): "Sociedad y deporte: análisis del deporte en la sociedad y su reflejo en los medios de comunicación en España". Universidade da Coruña.

ISASI VARELA, Antonio (2006): "La construcción del derbi futbolístico Real Madrid C. F. - F. C. Barcelona en el discurso periodístico de El País y La Vanguardia entre 1996 y 2004". Santiago de Compostela, Universidade de Santiago de Compostela.

KRIPPENDORFF, Klaus (2002): Metodología del análisis de contenido. Teoría y práctica. Barcelona, Paidós.

LLOPIS GOIG, Ramón (2008): "Identity, nation-state and football in Spain. The evolution of nationalist feelings in Spanish football", en Soccer \& Society, N ${ }^{\circ}$ 9(1). pp. 56-63.

MARTÍNEZ ALBERTOS, José Luis (2000): Curso general de redacción periodística. Madrid, Paraninfo.

MEÂN, Lindsey y HALONE, Kelby (2010): "Sport, Language, and Culture: Issues and Intersections", en Journal of Language and Social Psychology, N²9(3). pp. 253-260.

PANIAGUA SANTAMARÍA, Pedro (2006): "Deporte en televisión: el pseudoperiodismo como espectáculo", en Estudios sobre el mensaje periodístico, $\mathrm{N}^{\circ} 12$. pp. 185-193.

ROWE, David (2004): Sport, Culture and the Media. 2 Edition. Glasgow, McGraw Hill Education.

RUIZ, José Antonio (2009): Fútbol, pan y circo. La metáfora patriótico-deportiva de España. Madrid, Fragua.

TÚÑEZ LÓPEZ, Miguel, et al. (2010): "Nuevos entornos, nuevas demandas, nuevos periodistas", en Estudios sobre el Mensaje Periodístico, vol. 16, pp. 79-94.

VÁZQUEZ MONTALBÁN, Manuel (2005): Fútbol: Una religión en busca de un Dios. Barcelona, Debate. 